\title{
Supply Chain Flexibility Assessment by Multivariate Regression and Neural Networks
}

\author{
Ananda S Jeeva ${ }^{1}$ and William W Guo ${ }^{2}$ \\ ${ }^{1}$ School of Information Systems, Curtin Business School, Curtin University of Technology \\ PO Box U1987 Perth, Western Australia \\ a.jeeva@curtin.edu.au \\ $3^{3 *}$ Faculty of Arts, Business, Informatics and Education, Central Queensland University \\ North Rockhampton QLD 4702, Australia \\ w.guo@cqu.edu.au
}

\begin{abstract}
This paper compares two vastly different methods of analysis - multiple regression and neural networks, in supply chain flexibility assessment. Data of manufacturing firms evaluating their prominent suppliers were analysed by multiple regression and simulated using three-layer multilayer perceptron (MLP) neural networks. Our study shows that NN can accurately determine a supplier's flexibility capability within an error of $1 \%$ The incorporation of these two methods can lead to better understanding and dynamic prediction of supply chain flexibility for buyers.
\end{abstract}

Keywords: Neural network, supply chain, flexibility, multivariate regression.

\section{Introduction}

The current economic climate pushes businesses to be more competitive and thus leads to re-evaluating their supply chain and supplier strategies. This competitiveness requires organisations to be more effective, efficient and productive with lower margin for error. It is also pushing companies to work more strategically with their supply chains and partners [1]. Hence strategic supply partnership is becoming more critical and will determine the sustainability and competitiveness of companies in the future [2]. Flexible supply policies also help companies adapt quickly to changing market conditions and become more competitive [3]. Competitiveness takes many forms, and this paper focuses on the parts of the supply chain activities that inter-relate with suppliers and manufacturers in their operational aspects.

Supply chain operational aspects have many dimensions of functions and activities. Specific dimensions are selected and considered in this paper. These selected dimensions are related to the activities of the responding manufacturing firms with their most prominent suppliers. These dimensions are information exchange, level of supplier integration, product and delivery variations, logistics, and the organisation's capability. These dimensions are selected as they represent some of the principal components of published supply chain literature as proposed by Croom et al [4].

These selected dimensions are hypothesised to have the highest ability and agility to be flexible. This flexibility is related to changes in the external and internal environment. As the factors in the external environment change, these supply chain dimensions are also expected to change accordingly to maintain the same level of effi- 
ciency and effectiveness. Flexibility and agility have been promulgated as strategic responses to the stochastic economy [5,6]. Aprile et al [7] agree that flexibility is a complex and multi-dimensional concept and propose that it reflects the capacity to adapt to changing internal and external conditions. Therefore, flexibility is necessary to maintain the competitive edge.

From a practitioner's point of view flexibility and adaptability are very necessary and useful in practical applications. Flexibility is necessary in many dimensions of the supply chain. Flexibility in delivery dates, quantity and inventory replenishments buffer the impact of uncertainties. Practitioners desire a flexibility guideline with practical applications. The 2009 AMR Research [8] of the top 25 leading supply chain leader companies shows that agility, responsiveness, reliability and predictability were rated very highly.

Efficacy of supply chain flexibility is further promulgated by Khan et al [9] who provide empirical justification that the flexible distribution practices promote greater organisational performance. This paper focuses on the determinate factors of supply chain flexibility to predict performance by flexibility measures. We describe two systems that use statistical and artificial neural network techniques respectively for determining the flexibility of suppliers based on their selected elements. The data set used in this study was collected from 241 manufacturers commenting about their suppliers' flexibility based on five dimensions of supply activities. A comparison is also made between these two approaches in terms of assessing supply chain flexibility.

\section{Selected Supply Chain Flexibility Dimensions}

The five dimensions that this study focuses on are information exchange, supplier integration, product and delivery, logistics, and organisational strategy. These five dimensions were found to be the common set of supply chain flexibility dimensions in most research literature [10].

The information exchange (IE) investigates the elements of suppliers' information, such as sufficiency of information, reliability of information, timeliness, accuracy, easy of sending, amount of human intervention required, and connectivity and compatibility of information systems. Agarwal [11] concluded that flexibility in IT systems is a strategic feature that offers unique advantages for decision making, competitiveness and systems design.

The supplier integration (SI) activity takes into consideration of the operational elements of suppliers' inventory levels, such as delivery schedule changes, service level standard, costs, ease and time to switch suppliers. The supplier integration activities represent the amount of suppliers' ability to integrate into the manufacturing process. Palsson and Johansson [12] conclude that the greater the integration the greater the opportunities for improvements in the supply chain activities.

Material compatibility is an issue in manufacturing. Ball et al [13] state that these issues arise when there are a number of different component variants. Product and delivery (PD) elements include the suppliers' ability to design and deliver new components, to modify the product mix, to implement product design, and to modify com- 
ponents variability. This element relates to the suppliers' capability and capacity to adapt to consumer's changing demands.

Supplier logistics (SL) elements are the physical movement and handling of the procured products. It acts as the physical link between supplier and buyer enabling the flow of products, components and materials. The delivery speed and reduction of lead time is critical in the production process and customer satisfaction [14]. The degree of flexibility may be constrained by the logistics carrier's environment and operational framework. For example, the cost of fuel and transportation may decide the best choice for logistics operations and how flexible it is for the supplier to modify logistics arrangements [15]. Therefore, logistics plays a critical role in supply chain flexibility. They include the modification of routes, transportation, performance and product variability, and materials handling in terms of product attributes.

Organisational strategy (OS) indicates how well the internal structure of the manufacturing organisation can adapt to changing external environment in terms of the supply chain functions. They include business strategy, cost of implementation, and responsive actions. Fantasy et al [16] in their empirical study found that firms need to build flexibility dimensions to fit into their organisational strategies to deal with direct effects of flexibility on performance.

Manufacturing organisations' suppliers that have the most flexibility in the above mentioned activities and elements will be able to respond to their own internal stochastic environment as well as their suppliers' external environment. The flexibility attributes must be built into all the activities and elements of supply chain dimensions discussed above.

\section{Multiple Regression Analysis}

This study was based on finding out the extent of manufacturers' suppliers flexibility capability in five key supply chain dimensions. Scale items were developed using Churchill [17] paradigm. Items were developed from literature review. Q-sort technique was used to refine the survey items. Pilot surveys were conducted and subjected to validity and reliability tests. Data was collected on a seven point Likert scale. The higher the number; the higher the flexibility.

The final survey instrument used a self administered postal survey which received a response rate of $21.1 \%$. The survey was administered to senior managers in the roles of supply chain/procurement/logistics/manufacturing/purchasing managers in large manufacturing companies. The sampling frame was 1300 companies from different industries ranging from fabricated metal products to food and kindred products. The main respondent criteria were manufacturers with more than 70 employees and annual procurement value of more than AUD $\$ 10$ million.

We know that the higher the five selected dimensions; the higher the flexibility of a supplier, but the correlation between the flexibility and five selected dimensions is still an unknown function. This is because the quantification of the five dimensions is based on human judgment that varies from person to person; hence correspondingly the flexibility is somehow an estimation based on the five dimensions. 
Traditional model for measuring and analysing such problem is based on multiple regression, which is expressed as

$$
Y=A_{0}+A_{1} x_{1}+A_{2} x_{2}+\ldots+A_{m} x_{m}+e,
$$

where $Y$ is the dependent variable as a function of $m$ independent variables $x_{1}, x_{2}, \ldots$, $x_{m} ; A_{0}, A_{1}, \ldots, A_{m}$ are the coefficients that determine the contribution of each independent variable to the outcome; $e$ is the error component and supposed to be a small constant [18]. This expression can be rewritten as

$$
Y=y+e
$$

where $y=A_{0}+A_{1} x_{1}+A_{2} x_{2}+\ldots+A_{m} x_{m}$ is the deterministic portion of the model and can be used to estimate the output of this model if all $m+1$ coefficients can be determined using sufficient known points. In fact, the set of coefficients determined by known points can only be regarded as the best estimate of individual coefficients due to the existence of random errors contained in the observed points. Therefore, the deterministic portion of the model, $y$, is approximated by

$$
\mathrm{y} \approx a_{0}+a_{1} x_{1}+a_{2} x_{2}+\ldots+a_{m} x_{m}
$$

where $a_{0}, a_{1}, \ldots, a_{m}$ are the estimated coefficients for $A_{0}, A_{1}, \ldots, A_{m}$, respectively.

One way to determine those estimated coefficients is to use the method of least squares through minimising the sum of squares of the errors (SSE) of $n$ known points as

$$
\mathrm{SSE}=\sum_{i=1}^{n}\left(Y_{i}-y_{i}\right)^{2}=\sum_{i=1}^{n}\left[Y_{i}-\left(a_{0}+a_{1} x_{i 1}+a_{2} x_{i 2}+\ldots+a_{m} x_{i m}\right)\right]^{2} .
$$

Minimising SSE will be achieved through solving the following system of simultaneous linear equations.

$$
\frac{\partial \mathrm{SSE}}{\partial a_{j}}=0 . \quad(j=0,1, \ldots, m) .
$$

For $n$ known points, this should lead to the $(m+1)$ least-squares linear equations in $a_{0}, a_{1}, \ldots, a_{m}$ as

$$
\begin{aligned}
& n a_{0}+\left(\sum x_{i 1}\right) a_{1}+\left(\sum x_{i 2}\right) a_{2}+\ldots+\left(\sum x_{i m}\right) a_{m}=\sum y_{i} \\
& \left(\sum x_{i 1}\right) a_{0}+\left(\sum x_{i 1}^{2}\right) a_{1}+\left(\sum x_{i 1} x_{i 2}\right) a_{2}+\ldots+\left(\sum x_{i 1} x_{i m}\right) a_{m}=\sum x_{i 1} y_{i} \\
& \left(\sum x_{i 2}\right) a_{0}+\left(\sum x_{i 1} x_{i 2}\right) a_{1}+\left(\sum x_{i 2}^{2}\right) a_{2}+\ldots+\left(\sum x_{i 2} x_{i m}\right) a_{m}=\sum x_{i 2} y_{i} \\
& \vdots \\
& \left(\sum x_{i m}\right) a_{0}+\left(\sum x_{i 1} x_{i m}\right) a_{1}+\left(\sum x_{i 2} x_{i m}\right) a_{2}+\ldots+\left(\sum x_{i m}^{2}\right) a_{m}=\sum x_{i m} y_{i}
\end{aligned}
$$

Solving this system of linear equations should determine the $(m+1)$ coefficients that hence define a multiple regression model. 
Using statistical tools provided in MATLAB, we apply multiple regression to the 241 entries mapped between the five factors as inputs and the flexibility as output (Flex), which defines a multiple regression model shown below

$$
\text { Flex }=0.0643-0.8593 I E+0.6009 S I-0.9827 P D-0.8693 S L-0.9538 O S .
$$

This regression is significant with a coefficient of 0.9852 and a maximum error of just about $10 \%$. The accuracy of determining the flexibility of a supplier using this regression will be discussed later incorporating the results from the neural network simulation.

\section{Neural Network Simulation}

Neural networks have been widely used in investment prediction and financial analysis $[19,20]$. This paper attempts to use it in a supply chain management environment. The use of neural networks offers several advantages over traditional statistical methods. There are no restrictive assumptions as imposed by traditional methodologies. Neural networks use all available information while incorporating new information through a learning process. It updates the old output and learns from its experience. Therefore it is flexible and adaptable and can be used in a changing environment like stochastic economy. Supply chain management activities need to be flexible to respond to external environmental uncertainties.

The core of a neural network is actually an adaptive mathematical model that is capable of approximating any arbitrary unknown function constrained by training datasets. It has been proven that a three-layer multilayer perceptron (MLP) neural network can approximate any continuous function mapped from one finite-dimensional space to another by adjusting the number of nodes in the hidden layer [21]. Numerous cases of three-layer MLP applications have been successful in different fields [22-25]. Figure 1 illustrates the structure of a three-layer MLP with one hidden layer of $L$ nodes, a $p$-dimensional input vector $X$ and a $q$-dimensional output vector $Y$. The relationship between the input and output components for this MLP can be generally expressed as.

$$
y_{k}=\varphi\left(\sum_{j=1}^{L} v_{k j} \psi\left(\sum u_{j i} x_{i}\right)\right)
$$

where $\varphi$ and $\psi$ are the transfer functions; $u_{j i}$ denotes the input-to-hidden layer weights at the hidden neuron $j$; and $v_{k j}$ is the hidden-to-output layer weights at the output unit $k$.

The neural network process involves two phases - training the network with known datasets and testing the trained network using different known datasets for model generalisation. This is different from statistical approaches, in which the same data can be used for both model generation and evaluation.

In this study, the MLP model is constructed and trained using neural network tools in MATLAB ${ }^{\circledR}$ [26]. The Levenberg-Marquardt algorithm [27] is chosen to train the 
selected MLPs because this algorithm has been reported to be the fastest method for training moderate-sized feedforward neural networks [28].

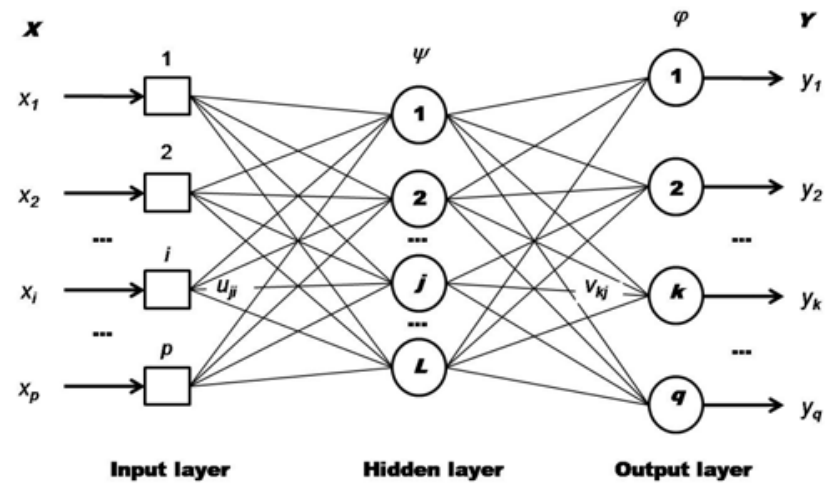

Fig. 1. Three layer multilayer perceptron (MLP) network

The 241 entries are compiled into two sets. The first set contains 207 (86\%) entries for training and the second set includes 34 (14\%) entries for testing. The log-sigmoidlinear combination is chosen as the transfer functions for our MLPs. The running of a number of nodes in the hidden layer shows that a 25-node hidden layer is good enough to achieve satisfactory training.

The test results for this 25-neuron MLP are tabulated in Table 1, along with the results for the regression model using the same test set. Both models show good quality for forecasting, but the MLP model is more accurate than the regression model, which can be clearly seen in Fig. 3 .

Table 1. Testing result of MLP and regression models

\begin{tabular}{llllll}
\hline & Test size & Correlation & MAE (\%) & SD (\%) & MAX (\%) \\
\hline MLP & 34 & 0.9999 & 0.15 & 0.24 & 0.88 \\
Regression & 34 & 0.9829 & 3.12 & 2.53 & 9.43 \\
\hline
\end{tabular}

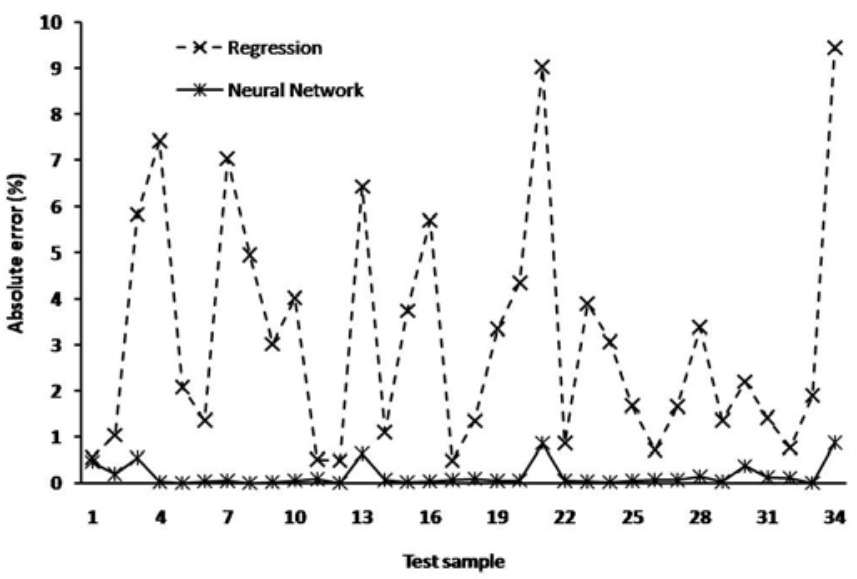

Fig. 2. Plots of relative errors 


\section{Discussion and Conclusion}

Although the multivariate regression defined in Relation (7) gives an analytical expression that is easier to understand, its performance for predicting a supplier's flexibility through its five attributes may result in an average error of $3 \%$ with a maximum error up to $10 \%$. This is still useful if the manufacturing scale is relatively small. However, if the scale of manufacturing is large, an error of $1 \%$ would mean a loss of millions of dollar in revenue.

The MLP model predicts the flexibility of a supplier with an average error of less than $0.2 \%$ and a maximum error within $1 \%$. The contrast of these two approaches is clearly illustrated in Fig. 3. Therefore, the neural network model outperforms the statistical model in quantitative simulation. This MLP model creates a dynamic discrete system that is able to approximate the nonlinear relation existing internally among the known entries. It is discrete because by feeding a new entry that falls in the range of the known domain to this neural system, it returns the closest approximation to the most relevant known entries in the domain. It is dynamic because when some new entries are added to the training data, this neural system is able to retrain the model in order to absorb these new entries into its coverage. This either enlarges the known applicable domain if the additional training data are beyond the previous range, or refines the local approximating mechanism if the additional training data are within the known domain.

In conclusion, the three-layered MLP is able to predict the supplier's flexibility with respect to its five attributes with a higher accuracy, compared with the multivariate regression analysis. However, multivariate regression is still useful for manufacturers with lesser number of suppliers and can give an understanding on the importance of individual attributes for the flexibility assessment through its analytical expression. This cannot be achieved by neural networks working like a black-box translator.

\section{References}

1. Wadhwa, S., Saxena, A., Chan, F.T.S.: Framework for flexibility in dynamic supply chain management. International Journal of Production Research 46, 1373-1404 (2008).

2. Min, S., Roath, A.S., Daugherty, P.J., Genchev, S.E., Chen, H., Arndt, A.D., Richey, R.G.: Supply Chain Collaboration: what's happening. The International Journal of Logistics Management 16, 237-256 (2005).

3. Xu, N.: Flexible supply policy with options and capacity constraints. Operations Research Letters 34, 508-516 (2006).

4. Croom, C., Romano, P., Giannakis, M.: Supply chain management: An analytical framework for critical review. European Journal of Purchasing and Supply Management 6, 6783 (2000).

5. Swafford, P. M., Ghosh, S., Murthy, N.: The antecedents of supply chain agility of a firm: scale development and model testing. Journal of Operations Management 24, 170-188 (2006).

6. Duclos, L.K., Vokurka, R.J., Lummus, R.R.: A concept model of supply chain flexibility. Industrial Management \& Data Systems 103, 446-456 (2003). 
7. Aprile, D., Garavelli, A. C., Giannoccaro, I.: Operations planning and flexibility in a supply chain. Production Planning \& Control 16, 21-31 (2005).

8. O’Marah, K., Hofman, D.: Top 25 supply chains. Supply Chain Management Review, October (2009) 12-19.

9. Khan, A.K., Bakkappa, B., Metri, B.A., Sahay, B.S.: Impact of agile supply chains' delivery practices on firms' performance: cluster analysis and validation. Supply Chain Management: An International Journal 14, 41-48 (2009).

10. Croom, C., Romano, P., Giannakis, M.: Supply chain management: an analytical framework for critical review. European Journal of Purchasing and Supply Management 6, 67$83(2000)$.

11. Agarwal, A.: Flexibility in information technology systems and organizational competitiveness. Global Journal of Flexible Systems Management 5, 35-38 (2005).

12. Palsson, H., Johansson, O.: Supply chain integration obtained through uniquely labeled goods: a survey of Swedish manufacturing industries. International Journal of Physical Distribution \& Logistics Management 39 28-46 (2009).

13. Ball, M.O., Chen, C.Y., Zhao, Z.Y.: Material compatibility constraints for make-to-order production planning. Operations Research Letters 31, 420-428 (2003).

14. Zhang, Q., Vonderembse, M.A., Lim, J.S.: Logistics flexibility and its impact on customer satisfaction. The International Journal of Logistics Management 16, 71-95 (2005).

15. Naim, M.M., PotterMason, A.T., R.J., Bateman, N.: The role of transport flexibility in logistics provision. The International Journal of Logistics Management 17, 297-311 (2006).

16. Fantazy, K.A., Kumar, V., Kumar, U.: An empirical study of the relationships among strategy, flexibility and performance in the supply chain context. Supply Chain Management: An International Journal 14, 177-188 (2009).

17. Churchill, G.A.: A paradigm for developing better measures of marketing constructs. Journal of Marketing Research 16, 64-73 (1979).

18. Mendenhall, W., Sincich, T.: Statistics for engineering and the sciences. Pearson Prentice Hall, Upper Saddle River (2007).

19. Yang, B., Li, L.X., Xu, J.: An early warning system for load risk assessment using artificial neural networks. Knowledge Based Systems 14, 303-306 (2001).

20. Kryzanowski, L., Galler, M., Wright, D.W.: Using artificial neural networks to pick stocks. Financial Analysts Journal 49, 21 (1993).

21. Hornik, K., Stinchcomb, M., White, H.: Multilayer feed forward networks are universal approximators. Neural Networks 2, 359-366 (1989).

22. Zhang, G.P., Keil, M., Rai, A., Mann, J.: Predicting information technology project escalation: A neural network approach. European Journal of Operational Research 146, 115-129 (2003).

23. Yan, H., Jiang, Y., Zheng, J., Peng, C., Li, Q.: A multilayer perceptron based medical decision support system for heart disease diagnosis. Expert Systems with Applications 30, 272-281 (2006).

24. Guo, W.W., Li, M.M., Whymark, G., Li, Z.X.: Mutual complement between statistical and neural network approaches for rock magnetism data analysis. Expert Systems with Applications 36, 9678-9682 (2009).

25. Guo, W.W.: Incorporating statistical and neural network approaches for student course satisfaction analysis and prediction. Expert Systems with Applications 37, 3358-3365 (2010).

26. Demuth, H., Beale, M., Hagan, M.: Neural network toolbox 5. The MathWorks, Natick (2007).

27. Marquardt, D.: An algorithm for least-squares estimation of nonlinear parameters. SIAM Journal on Applied Mathematics 11, 431-441 (1963).

28. Hagan, M.T., Demuth, H.B., Beale, M.H.: Neural network design. PWS Publishing, Boston (1996). 